Revista de Matemática: Teoría y Aplicaciones 2(2): 9-16 (1995)

\title{
ESTUDIO EN TIEMPO DISCRETO DE LOS PROCESOS PUNTUALES DE INCREMENTOS CONDICIONALEMENTE INDEPENDIENTES
}

\author{
Jaime Lobo Segura ${ }^{1}$
}

\begin{abstract}
Resumen
A partir de resultados obtenidos sobre la representación binaria de los procesos puntuales en tiempo continuo, se obtiene, bajo hipótesis de separabilidad, un análogo en tiempo discreto (procesos de incrementos casi condicionalmente independientes) de los procesos puntuales de incrementos condicionalmente independientes. El estudio de la función laplaciana condicionada del proceso en tiempo discreto permite deducir bajo ciertas condiciones de continidad una fórmula explícita para la respectiva del proceso puntual en tiempo continuo.
\end{abstract}

Palabras clave: procesos puntuales en tiempo continuo (tiempo discreto); hipótesis de no explosión y separabilidad; procesos puntuales de incrementos condicionalmente independientes (casi condicionalmente independientes); función laplaciana condicionada; hipótesis de continuidad en probabilidad condicionada; ley de Poisson doblemente estocástica

\section{Introducción}

El autor de este trabajo esbozó una teoría en [4] sobre los llamados procesos puntuales en tiempo discreto (ppd). Se trata de una clase especial de procesos estocásticos en espacios finitos, y que resultan ser el análogo discreto de los procesos puntuales en el sentido clásico (tiempo continuo). En términos del análisis no standard se define así: dado un casi intervalo $\Pi$ de $[0, t], N$ es ppd sobre $\Pi$ si cumple la propiedad fundamental de que los incrementos $d N_{t}$ de $N$ sobre los puntos de $\Pi$ son variables de Bernoulli con valores en $\{0,1\}$. Se demostró que todo proceso puntual $N$ sobre $[0, t]$ no explosivo y standard posee un ppd aledaño $N^{\prime}$, en el sentido siguiente: $N_{\Pi}=N^{\prime}$ salvo en un evento de probabilidad infinitesimal ( $N_{\Pi}$ designa la restricción de $N$ al conjunto de índices $\left.\Pi\right)$. Se plantea entonces el desarrollo de un programa finitista para los procesos puntuales basado en el estudio de los ppd.

\footnotetext{
${ }^{1}$ Escuela de Matemática, Universidad de Costa Rica
} 
Siguiendo esta línea de ideas me propongo aquí estudiar una clase de procesos puntuales en tiempo continuo denominados de incrementos condicionalmente independientes (II condicionales). Dados un espacio $(\Omega, \mathcal{A}, P)$ y una subtribu $A$ de $\mathcal{A}$ (tribu condicionante), un proceso puntual $N$ es II condicional con respecto a $A(\mathrm{II} / / A)$ si cumple la condición de que los incrementos de $N$ sobre intervalos disjuntos son condicionalmente independientes con respecto a $A$ (se supone además que existe una versión regular $P_{A}$ de la ley condicional de $N$ con respecto a $A$, es decir el de una aplicación $P_{A}$ de $\Omega$ en el conjunto de probabilidades sobre $(\Omega, \mathcal{A})$ tal que para toda variable $Y \sigma(N)$-integrable se tenga:

$$
E(Y \mid A)(w)=\int Y\left(w^{\prime}\right) P_{A}\left(w ; d w^{\prime}\right) \text { casi siempre. }
$$

Si la tribu condicionante es $\{\Omega, \phi\}$ se habla de proceso puntual de incrementos independientes (II).

Una versión discreta de la propiedad II condicional fue ofrecida en [4], pudiendo en este caso ser formulada en términos de filtraciones : siendo $N$ un ppd sobre $\Pi$ y $A$ una subtribu de $(\Omega, P)$, y siendo $F=\left(F_{t}\right)_{t \in \Pi}$ la filtración generada por $N$ y $A: F_{t}=\sigma\left(N_{s}, s \in \Pi, s \leq t\right) V A$, es II //A si cumple para todo $t \in \Pi: E_{t}\left(d N_{t}\right)=E_{A}\left(d N_{t}\right),\left(E_{t}(\cdot)\right.$ y $E_{A}(\cdot)$ designan las esperanzas condicionales con respecto a $F_{t}$ y $A$ respectivamente).

Lo primero que estableceremos es que para los procesos puntuales II condicionales en tiempo continuo es posible, bajo condiciones adecuadas sobre la tribu condicionante $A$, encontrar para cada ppd $N^{\prime}$ aledaño a éste una subtribu finita de $A$ tal que $N^{\prime}$ goza de una propiedad que llamaré de incrementos casi condicionalmente independientes (ICI condicionales). Esta clase de procesos generaliza la de los procesos ppd II condicionales. Se estudia luego una aproximación para la función laplaciana de los incrementos de un ICI condicional. Finalmente en la sección 3 se determina la ley de los incrementos de un II condicional en tiempo continuo a partir de la laplaciana de su ppd ICI condicional asociado, que bajo ciertas condiciones de regularidad resulta ser la de un proceso de Poisson doblemente estocástico.

El marco formal adoptado para el análisis no standard es el de la "Internal Set Theory"(IST), cuya exposición general puede hallarse en [5]. Se usará la terminología y notación standard siguientes : un número real $x$ es infinitesimal si $|x| \leq a$ para todo $a>0$ standard. Es llamado ilimitado si $|x|>a$ para todo real $a$ standard. Si $x, y$ son reales denotamos: $x \approx y$ si $x-y$ es infinitesimal, $x \approx \infty$ si $x$ es ilimitado, $x \ll \infty$ si $x$ no es ilimitado positivo. Refiero a [5] para los aspectos generales de la teoría de espacios finitos con métodos de la IST.

\section{Proceso ICI condicional asociado a un II condicional}

Sean dados un espacio de probabilidades finito $\Omega, P$ ), un casi intervalo $\Pi$ de $[0, t]$, y una partición $A$ de $\Pi$. Un ppd $N$ sobre $\Pi$ se dirá de incrementos casi condicionalmente independientes con respecto a $A(\mathrm{ICI} / / A)$ si para la filtración $F=\left(F_{t}\right) t \in \Pi$ generada por $N$ y $A$ se cumple:

a) c.s. $N_{t}$ es limitado, 
b) $\sum_{t \in \Pi}\left\|E_{t}\left(d N_{t}\right)-E_{A}\left(d N_{t}\right)\right\|_{2} \approx 0$.

Los procesos ICI condicionales generalizan los II condicionales. En efecto, si la condición b) se cumple además con igualdad se debe tener que para todo $t \in \Pi: \| E_{t}\left(d N_{t}\right)-$ $E_{A}\left(d N_{t}\right) \|_{2}=0$, y siendo $\Omega$ finito, necesariamente $E_{t}\left(d N_{t}\right)=E_{A}\left(d N_{t}\right)$.

Consideramos un proceso puntual $N$ sobre $[0, t]$ standard y no explosivo, definido en espacio $(\Omega, \mathcal{A}, P)$ standard, y que es II condicional con respecto a una subtribu standard $A$ de $\mathcal{A}$. Sea $N^{\prime}$ un ppd aledaño a $N$ sobre un casi intervalo $\Pi$ de $[0, t]$, que satisface la condición: $\forall t \in \Pi, d N_{t}^{\prime}$ es $d N_{t}--$ medible. Esta última condición se logra siempre cumplir modificando un poco la construcción dada en [4]. Para ello considere $\Pi$ el casi intervalo definido en el teorema 1 en [4] y defina $N^{\prime}$ por $d N_{t}^{\prime}=\operatorname{mín}\left(d N_{t}, 1\right)=1_{\left\{d N_{t} \geq 1\right\}}$, y es claro que $N^{\prime}$ así definido es un ppd aledaño a $N$ cumpliendo la condición anterior. Tenemos :

Teorema 1 Bajo las condiciones anteriores y la hipótesis de que A es separable, existe una subtribu finita $A^{\prime}$ de $A$ tal que, en el espacio finito generado por $N^{\prime}$ y $A^{\prime}, N^{\prime}$ es un ICI condicional con respecto a $A^{\prime}$. Si además $A$ es ella misma finita se puede tomar $A^{\prime}=A y$ $N^{\prime}$ es un II condicional con respecto a $A^{\prime}$.

Necesitaremos antes del lema siguiente :

Lema 1 Bajo las hipótesis del teorema 1, existe una subtribu finita $A^{\prime}$ de $A$ tal que:

$$
\sum_{t \in \Pi}\left\|E_{A}\left(d N_{t}^{\prime}\right)-E_{A^{\prime}}\left(d N_{t}^{\prime}\right)\right\|_{2} \approx 0
$$

DEmonstración: Siendo $A$ una tribu separable es la tribu engendrada por una sucesión creciente de $\left(\mathcal{A}_{\backslash}, n \in N\right)$ de subtribus finitas de $\mathcal{A}$ en $(\Omega, \mathcal{A}, P)$. Se sabe que para todo $Y \in$ $L_{2}(\Omega, \mathcal{A}, P), \lim _{n \rightarrow \infty} E_{\mathcal{A}_{n}}(Y)=E_{A}(Y)$ en $L_{2}$, (ver por ejemplo Neveu [7]). En particular para $t$ en $\Pi$, el incremento $d N_{t}^{\prime}$ siendo una v.a. binaria es cuadrado integrable y entonces :

$$
\forall \varepsilon>0 \text {, existe } m \in N \text { tal que si } p>m:\left\|E_{A}\left(d N_{t}^{\prime}\right)-E_{A_{p}}\left(d N_{t}^{\prime}\right)\right\|_{2}<\varepsilon \text {. }
$$

Escoja $\varepsilon$ infinitesimal suficientemente pequeño de tal manera que $\varepsilon|\Pi| \approx 0 \mathrm{y}$, siendo $\Pi$ finito, un $p$ entero suficientemente grande para que

$$
\left\|E_{A}\left(d N^{\prime} t\right)-E_{\mathcal{A}_{p}}\left(d N^{\prime} t\right)\right\|_{2}<\varepsilon, \forall t \in \Pi .
$$

Entonces : $\sum_{t \in \Pi}\left\|E_{A}\left(d N_{t}^{\prime}\right)-E_{\mathcal{A}_{p}}\left(d N_{t}^{\prime}\right)\right\|_{2} \approx 0$.

Basta entonces escoger $A^{\prime}$ como $\mathcal{A}_{p}$.

Demonstración(teorema 1): Puesto que $N$ es no explosivo su proceso $N^{\prime}$ es c.s. limitado, de donde la propiedad a) de la definición de un ICI condicional.

Sea $A^{\prime}$ la tribu definida en el lema 1 . Sean $F=\left(F_{t}\right)_{t \in \mathbb{R}}$ y $F^{\prime}=\left(F_{t}^{\prime}\right)_{t \in \Pi}$ las filtraciones generadas respectivamente por $N$ y $A, N^{\prime}$ y $A^{\prime}$. Por $E_{t^{\prime}}(\cdot)$ denotamos la esperanza condicional con respecto a $F_{t}^{\prime}$. Para cada $t \in \Pi, d N_{t}^{\prime}$ es función de $d N_{t}$ y por lo tanto la hipótesis 
de independencia condicional implica que: $E_{A}\left(d N_{t}^{\prime}\right)=E_{t}\left(d N_{t}^{\prime}\right)$. La propiedad del doble condicionamiento implica además que:

$$
E_{t^{\prime}}\left(d N^{\prime}\right)=E_{t^{\prime}}\left(E_{t}\left(d N_{t}^{\prime}\right)\right), E_{A^{\prime}}\left(d N_{t}^{\prime}\right)=E_{t^{\prime}}\left(E_{A^{\prime}}\left(d N_{t}^{\prime}\right)\right)
$$

y por lo tanto:

$$
E_{t^{\prime}}\left(d N_{t}^{\prime}\right)-E_{A^{\prime}}\left(d N_{t}^{\prime}\right)=E_{t^{\prime}}\left(E_{A}\left(d N_{t}^{\prime}\right)-E_{A^{\prime}}\left(d N_{t}^{\prime}\right)\right)
$$

De esta última relación, del hecho de que la esperanza condicional reduce la norma y del resultado del lema 1 se deduce :

$$
\sum_{t \in \Pi}\left\|E_{t}\left(d N_{t}^{\prime}\right)-E_{A^{\prime}}\left(d N_{t}^{\prime}\right)\right\|_{2} \approx 0
$$

con igualdad si $A$ es ella misma finita. Esto demuestra para $N^{\prime}$ la propiedad b) de la definición de un ICI condicional y la propiedad II condicional en el caso de ser $A$ finita.

\section{Función laplaciana condicionada de los incrementos de un ICI condicional}

Se consideran un espacio de probabilidades finito $\Omega, P$ ) y un casi intervalo $\Pi$ de $[0, t]$. En el resto de esta sección se designa por $N$ un ppd sobre $\Pi$, ICI con respecto a $A$. Como anteriormente se denota por $F=\left(F_{t}\right)_{t \in \Pi}$ la filtración generada por $N$ y $A$. Sea $N^{p}$ el $F$-compensador previsible de $N$. Gracias al lema 4 del apéndice y la propiedad a) de $N$, su compensador previsible con respecto a $F$ es c.s. limitado. Por otro lado para todo $t \in \Pi$ se cumple:

$$
\left|N_{t}^{p}-E_{A}\left(N_{t}\right)\right| \leq \sum_{t \in \Pi}\left|E_{t}\left(d N_{t}\right)-E_{A}\left(d N_{t}\right)\right|
$$

y de la definición de un ICI: $\left\|\operatorname{máx}_{t \in \Pi}\left\{\left|N_{t}^{p}-E_{A}\left(N_{t}\right)\right|\right\}\right\|_{2} \approx 0$. Deducimos entonces:

Lema 2 Para todos $s, t, s<t$ en $\Pi$, se tiene: c. $s . E_{A}(N t-N s)$ es limitado.

El teorema que sigue ofrece una aproximación para la función laplaciana condicionada : $\lambda \rightarrow E_{A}\left(e^{-\lambda\left(N_{t}-N_{s}\right)}\right)$, de los incrementos de $N$.

Teorema 2 Para $\lambda \geq 0$, se tiene:

$$
\left\|\operatorname{máx}_{t \in \Pi, t \geq s} \mid E_{A}\left(e^{-\lambda\left(N_{t}-N_{s}\right)}\right)-\prod_{u: s \leq u<t}\left(1+\left(e^{-\lambda}-1\right) E_{A}\left(d N_{u}\right)\right)\right\|_{2} \approx 0
$$

Esta relación se cumple con igualdad si $N$ es además un II condicional.

DEMONSTRACión: Se define el proceso $Y$ en $\{t \in \Pi: t \geq s\}$ por $Y_{t}=E_{s}\left(e^{-\lambda\left(N_{t}-N_{s}\right)}\right)$. Siendo $d N_{t}$ con valores en $\{0,1\}$ se tiene:

$$
e^{-\lambda N_{t}+d t}-e^{-\lambda N_{t}}=e^{-\lambda N_{t}}\left(e^{-\lambda}-1\right) d N_{t}
$$


Por lo tanto $d Y_{t}=E_{s}\left(e^{-\lambda\left(N_{t}-N_{s}\right)}\left(e^{-\lambda}-1\right) d N_{t}\right)$. Como $E_{s}\left(E_{t}(\cdot)\right)=E s(\cdot)$ lo anterior se escribe:

$$
d Y_{t}=\left(e^{-\lambda}-1\right) E_{s}\left(e^{-\lambda\left(N_{t}-N_{s}\right)} \cdot E_{t}\left(d N_{t}\right)\right)=\left(e^{-\lambda}-1\right) E_{s}\left(e^{-\lambda\left(N_{t}-N_{s}\right)} \cdot d N_{t}^{p}\right) .
$$

Sumamos y restamos la cantidad $\left(e^{-\lambda}-1\right) E_{s}\left(e^{-\lambda\left(N_{t}-N_{s}\right)} E_{A}\left(d N_{t}\right)\right)$ en esta relación para obtener :

$$
d Y_{t}=\left(e^{-\lambda}-1\right) E_{s}\left(e^{-\lambda\left(N_{t}-N_{s}\right)} E_{A}\left(d N_{t}\right)\right)+\varepsilon_{t}, Y_{s}=1
$$

siendo $\varepsilon_{t}=\left(e^{-\lambda}-1\right) E_{s}\left(e^{-\lambda\left(N_{t}-N_{s}\right)}\left(d N_{t}^{p}-E_{A}\left(d N_{t}\right)\right)\right.$. Dado que $E_{s}\left(E_{A}(\cdot)\right)=E_{A}(\cdot)$ la relación puede escribirse como:

$$
d Y_{t}=\left(e^{-\lambda}-1\right) Y_{t} E_{A}\left(d N_{t}\right)+\varepsilon_{t}, Y_{s}=1
$$

Como la esperanza condicional reduce la norma se tiene:

$$
\left\|\varepsilon_{t}\right\|_{2} \geq \|\left(e^{-\lambda}-1\right) e^{-\lambda\left(N_{t}-N_{s}\right)}\left(d N_{t}^{p}-E_{A}\left(d N_{t}\right)\left\|_{2} \geq\right\| d N_{t}^{p}-E_{A}\left(d N_{t}\right) \|_{2}\right.
$$

Se deduce que la sucesión $\varepsilon=\left(\varepsilon_{t}\right)_{t \in \Pi, t \geq s}$ cumple $\sum_{t \in \Pi, t \geq s}\left\|\varepsilon_{t}\right\|_{2} \approx 0$.

El conjunto $\Pi$ siendo finito, la relación (1) puede verse como una relación recurrente lineal de primer orden en la sucesión $\left(Y_{t}\right)_{t \in \Pi, t \geq s}$. De la teoría elemental de estas sucesiones podemos obtener explícitamente :

$$
Y_{t}=\sum_{u: s \leq u<t}\left(1+b_{u}\right)+\sum_{u: s \leq u<t} \varepsilon_{u} \cdot \prod_{u^{\prime}: u<u^{\prime}<t}\left(1+b_{u^{\prime}}\right)
$$

donde $b_{u}=\left(e^{-\lambda}-1\right) E_{A}\left(d N_{u}\right)$. Tomando esperanza condicional con respecto a $A$ se cumple para todo $t \in \Pi, t>s$ :

$$
\left|E_{A}\left(Y_{t}\right)-\prod_{u: s \leq u<t}\left(1+b_{u}\right)\right| \leq \sum_{t \in \Pi}\left|E_{A}\left(\varepsilon_{t}\right)\right|
$$

y se obtiene la relación del teorema tomando la norma en esta desigualdad y usando el hecho de que $E_{A}\left(E_{s}(\cdot)\right)=E_{A}(\cdot)$.

En el caso particular de un ppd II condicional la sucesión $\varepsilon$ es nula, y las relaciones anteriores se obtienen además con igualdad.

Corolario 1 Si $\lambda \geq 0$, y c.s.: máx $_{t \in \Pi}\left|E_{A}\left(d N_{t}\right)\right| \approx 0$, se tiene para $t>s$ :

$$
\left\|E_{A}\left(e^{-\lambda\left(N_{t}-N_{s}\right)}\right)-\exp \left(\left(e^{-\lambda}-1\right) E_{A}\left(N_{t}-N_{s}\right)\right)\right\|_{2} \approx 0 .
$$

DEMONSTRACión: Examinemos un desarrollo asintótico para $\prod_{u: s \leq u<t}\left(1+b_{u}\right)$, donde $b_{u}=$ $\left(e^{-\lambda}-1\right) E_{A}\left(d N_{u}\right)$. Por un lado $\log \left(1+b_{u}\right) \sim b_{u}$ cada vez que $b_{u} \neq 0$, y en este caso tienen el mismo signo. De la hipótesis:

$$
\text { c.s. : } \operatorname{máx}_{s \leq u<t}\left|b_{u}\right| \leq \max _{s \leq u<t}\left|E_{A}\left(d N_{u}\right)\right| \approx 0
$$


por lo que, c.s.:

$$
\log \left(\prod_{u: s \leq u<t}\left(1+b_{u}\right)\right)=\prod_{u: s \leq u<t} \log \left(1+b_{u}\right) \sim \sum_{u: s \leq u<t}\left(e^{-\lambda}-1\right) E_{A}\left(d N_{u}\right)
$$

(la última relación asintótica se deriva del teorema 5.3, [6]).

Tomando exponencial en esta relación :

$$
\prod_{u: s \leq u<t}\left(1+b_{u}\right) \sim \exp \left(\left(e^{-\lambda}-1\right) E_{A}\left(N_{t}-N_{s}\right)\right), \text { c.s. }
$$

La expresión del miembro derecho en $\sim$ es positiva menor que 1 , no infinitesimal según el lema 2. Del teorema 5.2 en [6] se sigue que para $\lambda \geq 0$ :

$$
\prod_{u: s \leq u<t}\left(1+b_{u}\right) \approx \exp \left(\left(e^{-\lambda}-1\right) E_{A}\left(N_{t}-N_{s}\right)\right), \text { c.s. }
$$

En esta relación ambas variables son Loeb-Nelson integrables pues son acotadas por 1 y gracias al teorema de Lebesgue (versión Loeb-Nelson, teorema 8.2, [6]) son infinitamente cercanas en la métrica $L_{2}$. Pero según el teorema 2 la variable del miembro izquierdo es infinitamente cercana en $L_{2}$ a $E_{A}\left(e^{-\lambda\left(N_{t}-N_{s}\right)}\right)$, de donde el resultado.

\section{Aplicación : ley de los incrementos de un II condicional en tiempo continuo}

Como aplicación de los resultados obtenidos en las secciones 1 y 2 , obtendremos una fórmula explícita para la ley de los incrementos de un II condicional en tiempo continuo:

Teorema 3 Sea $N$ un proceso puntual en tiempo continuo, II condicional con respecto a A, siendo A separable. Suponemos además que $N$ es c.s. continuo en probabilidad con respecto a una versión regular $P_{A}$ de la ley condicional de $N$ con respecto a A. Entonces para todos $v, u \in[0, t], v>u$ :

$$
E_{A}\left(e^{-\lambda\left(N_{v}-N_{u}\right)}\right)=\exp \left(\left(e^{-\lambda}-1\right) E_{A}\left(N_{v}-N_{u}\right)\right)
$$

es decir, la ley de $N$ es de Poisson doblemente estocástica.

Para deducir el teorema 1, basta por transfer suponer que $N$ es standard. Existe según el teorema 1 de la sección 1 un proceso $N^{\prime}$ ICI condicional con respecto a $A^{\prime}$, aledaño a $N$ sobre un casi intervalo $\Pi$ de $[0, t]$. Necesitamos la propiedad de $N^{\prime}$ siguiente :

Lema 3 c.s.: $\operatorname{máx}_{t \in \Pi}\left|E_{A^{\prime}}\left(d N_{t}^{\prime}\right)\right| \approx 0$.

DEMONSTRACión: Consideremos una sucesión standard $\Pi_{n}$ de particiones de $[0, t]$ tal que $\Pi=\Pi_{\nu}$ para un $\nu$ ilimitado (ver [4] sobre la construcción de $\Pi$ ). La hipótesis de continuidad en probabilidad con respecto a $P_{A}$ significa que:

$$
\operatorname{máx}_{s \in \Pi_{n}} P_{A}\left(d N_{s} \geq 1\right) \rightarrow 0, \text { en probabilidad cuando } n \rightarrow \infty .
$$


Pero la sucesión de variables ( $\left.\operatorname{máx}_{s \in \Pi_{n}} P_{A}\left(d N_{s} \geq 1\right)\right)_{n \in N}$ es standard. De la caracterización no standard de la convergencia en probabilidad se deduce que existe $\lambda$ infinitesimal tal que:

$$
P\left(\operatorname{máx}_{s \in \Pi} P_{A}\left(d N_{s} \geq 1\right) \geq \lambda\right) \approx 0 .
$$

Ahora bien, $P_{A}\left(d N_{s} \geq 1\right)=E_{A}\left(d N_{s}^{\prime}\right)$, y de la relación obtenida en el lema 1 se obtiene: $P\left(\operatorname{máx}_{s \in \Pi} E_{A}^{\prime}\left(d N_{s}^{\prime}\right) \geq \lambda\right) \approx 0$, que es lo que se requería.

DEMONSTRACIÓN(teorema 3): Dados dos reales standard fijos $u, v$ de $[0, t]$ se puede suponer sin pérdida de generalidad que $u, v \in \Pi$. Entonces las variables $e^{-\lambda\left(N^{\prime} v-N^{\prime} u\right)}, e^{-\lambda(N v-N u)}$ coinciden salvo en evento infinitesimal, y siendo acotadas por 1 , son infinitamente cercanas en $L_{2}$. La esperanza condicional retracta la norma, por lo que $X=E_{A^{\prime}}\left(e^{-\lambda\left(N^{\prime} v-N^{\prime} u\right)}\right)$ y $Y=E_{A^{\prime}}\left(e^{-\lambda(N v-N u)}\right)$ son infinitamente cercanas en $L_{2}$. Son también infinitamente cercanas en $L_{2}$ :

- $X$ y $\exp \left(\left(e^{-\lambda}-1\right) E_{A^{\prime}}\left(N_{t}^{\prime}-N_{s}^{\prime}\right)\right)$ según el corolario 1 y el lema 3 .

- $\exp \left(\left(e^{-\lambda}-1\right) E_{A^{\prime}}\left(N_{t}^{\prime}-N_{s}^{\prime}\right)\right)$ y $\exp \left(\left(e^{-\lambda}-1\right) E_{A}\left(N_{t}^{\prime}-N_{s}^{\prime}\right)\right)$ según el lema 1 .

- $E_{A}\left(e^{-\lambda\left(N_{v}-N_{u}\right)}\right)$ y $Y$, por definición de la tribu $A^{\prime}$ (ver prueba del teorema 1 ) y del hecho que $E_{A}\left(e^{-\lambda\left(N_{v}-N_{u}\right)}\right)$ es variable standard cuadrado integrable.

De todo lo anterior tenemos que $E_{A}\left(e^{-\lambda\left(N_{v}-N_{u}\right)}\right)$ y $\exp \left(\left(e^{-\lambda}-1\right) E_{A}\left(N_{t}-N_{s}\right)\right)$ son infinitamente cercanas en $L_{2}$. Pero ambas variables son a su vez standard por lo que necesariamente son iguales en la métrica $L_{2}$, es decir casi siempre iguales.

Se obtiene como corolario del teorema 4 la caracterización clásica de los procesos de Poisson :

Corolario 2 Si $N$ es un II en tiempo continuo, continuo en probabilidad, la ley de sus incrementos son del tipo Poisson.

Observaciones finales

Se desprende del estudio efectuado en este trabajo el interés por desarrollar una teoría de procesos puntuales en tiempo discreto. Por un lado la estructura de los ppd es obviamente mucha más simple que la de los procesos clásicos, prueba de ello es el desarrollo presentado en la sección 2 donde se hizo patente el uso de las técnicas del "cálculo estocástico"en tiempo discreto. Por otro lado al contar con una adecuada versión discreta de una clase de procesos en tiempo continuo (en este caso los II condicionales) se ofrece la posibilidad deducir sus propiedades en terminos clásicos.

Los procesos puntuales de incrementos condicionalmente independientes en tiempo continuo han sido objeto de estudio por varios autores, estudiando también la forma de su ley. Guikhman y Skorohod [1] estudian los II continuos en probabilidad estableciendo el carácter poissoniano de su ley con razonamientos clásicos bastante complejos. Más generalmente Grigelionis [2] estudia los II condicionales, sin la hipótesis de la continuidad en probabilidad, y deduce una fórmula general para la laplaciana condicionada de los incrementos. Debe sin embargo recurrir a las técnicas generales del cálculo estocástico en tiempo continuo y en particular a las propiedades del llamado compensador previsible del 
proceso, en el sentido de la teoría general de procesos (Jacod desarrolló esta teoría en toda generalidad en [3]). Lo que he demostrado aquí es que es posible rescatar estas técnicas del cálculo estocástico pero solamente para los procesos en tiempo discreto, lo que evita muchas de las dificultades conceptuales presentes en la teoría general de procesos.

Es interesante preguntarse de qué manera con los métodos finitos se pueden generalizar los resultados obtenidos con hipótesis menos fuertes sobre los procesos. Por ejemplo se podría examinar si la hipótesis de separabilidad sobre la tribu condicionante en la definición de los procesos II condicionales es realmente necesaria. También se puede tratar de extender el resultado del teorema 3 sin suponer la hipótesis de continuidad en probabilidad condicionada. Estos problemas conducirían ciertamente a replantear las nociones sobre los ppd y a reformular los resultados que se han expuesto en este trabajo.

\section{Apéndice}

En un espacio finito $(\Omega, P)$ y dado un casi intervalo $\Pi$ que contiene al origen, se considera un proceso puntual $N$ en tiempo discreto sobre $\Pi$. Se estableció en [4] que, en general, dada una filtración $F=\left(F_{t}\right)_{t \in \Pi}$ sobre $(\Omega, P)$ para la cual $N$ es $F$-adaptado, es posible definir el $F$-compensador previsible de $N$, denotado por $N^{p}$, por $d N_{t}^{p}=E_{t}\left(d N_{t}\right)$ y $N_{0}^{p}=0$. Varias de sus propiedades fueron estudiadas y en particular la llamada propiedad fundamental de integración. Incluimos una más que no fue incluida en ese trabajo:

Lema $4 N$ es casi siempre limitado sii $N^{p}$ también lo es.

DEmonstración: Supongamos $N$ casi siempre limitado. Par cada $n$ entero definimos un proceso $X F$-adaptado por: $X_{t}=1$ si $N_{t}<n, X_{t}=0$ en el caso contrario. Entonces $X * N_{t}=\sum_{s<t} X_{s} d N_{s}=N_{t}$ si $N_{t}<n, n$ si $N_{t} \geq n$, es decir: $X * N \leq n$ en $\Pi$. Por la propiedad fundamental del compensador previsible: $E\left(X * N_{t}\right)=E\left(X * N_{t}^{p}\right) \leq n$. Entonces si $n$ es limitado $X * N_{t}^{p}$ es c.s. limitada. En $\left\{N_{t}<n\right\}, X=1$ en todo $\Pi$ y $X * N_{t}^{p}=N_{t}^{p}$. Como además para cada $\varepsilon \gg 0$ existe $n$ limitado tal que $P\left\{N_{t} \geq n\right\}<\varepsilon$, deducimos que $N^{p}$ es casi siempre limitado. La prueba en el otro sentido es análoga invirtiendo los roles de $N$ y $N^{p}$.

\section{Referencias}

[1] Guikhman \& Skorohod (1980) Introduction à la Théorie des Processus Aléatoires. (Traducción francesa) Mir, Moscú.

[2] Grigelionis (1975) "Characterization of point process with conditionally independent increments", Litov. Mat. Sbor., XV(4).

[3] Jacod (1975) "Multivariate point process : predictable projection, Radon-Nikodym derivatives, representation of martingales", Z. Wahrsch. Verw. Geb. 31(3).

[4] Lobo, J. (1995) "Una noción de proceso puntual en tiempo discreto", Revista de Matemática: Teoría y Aplicaciones, 2(1): 17-25. 
[5] Nelson (1977) "Internal Set Theory : a new approach to nonstandard analysis", Bulletin of the American Mathematical Society, 83(6).

[6] Nelson, E. (1987) Radically Elementary Probability Theory. Princeton University Press, New Jersey.

[7] Neveu, J. (1972) Martingales à Temps Discret. Masson, Paris. 